\title{
Small Round Blue Cell Tumor of Seminal Vesicle in a Young Patient
}

\author{
Adriano A. de Paula, Adriano R. Maltez, Eliane D. Mota \\ Sections of Oncological Urology and Pathology, Araujo Jorge Hospital, Goiania, Goias, Brazil
}

\begin{abstract}
Seminal vesicle tumor is a rare disease with unclear origin. Generally, it is presented as a pelvic mass that can be detected by sonography and digital rectal exam. The authors report a 25 -year-old patient with a pelvic mass which the magnetic resonance and surgical specimen reveal a seminal vesicle tumor. Immunohistochemical findings favored a primitive neuroectodermal tumor of the seminal vesicle. Herein, the treatment, histological and histochemical findings of this entity are discussed.
\end{abstract}

Key words: seminal vesicles; neuroectodermal tumors; urogenital neoplasms

Int Braz J Urol. 2006; 32: 566-9

\section{INTRODUCTION}

Seminal vesicle tumors are rare malignancies, usually carcinomas and are generally presented as a retrovesical mass that can be detected by digital rectal examination and sonography (1). Small round blue cell tumors (SRBCT) is a group of neoplasms that share a common and unique chromosomal translocation and include the primitive neuroectodermal tumor (PNET) and the Ewing's sarcoma family. PNET typically develops in pediatric population, arising from the thoracic region, but genitourinary involvement in adulthood is uncommon.

\section{CASE REPORT}

A 25-year-old man underwent abdominal sonography due to epygastric pain which showed a complex-cystic mass with 5,0x 4,9x $5,5 \mathrm{~cm}$ in the topography of seminal vesicles. A pelvic computerized tomography confirmed a solid retrovesical lesion. The patient declined an ultrasound guided trans-rectal biopsy and never returned during a 12 month-period. After this period he returned complaining of rectal stricture, and lower urinary obstructive symptoms.

Pelvic magnetic resonance showed a $17 \mathrm{~cm}$ retrovesical mass (Figure-1) and a trans-rectal ultrasound guided biopsy revealed small round cells for which immunohistochemical findings suggested a primitive neuroectodermal tumor diagnosis. Two cycles of neo-adjuvant chemotherapy consisting of vincristine, cyclophosphamide, doxorubicin, ifosfamide and etoposide were performed, resulting in no radiological response.

Workup for metastasis was negative and the patient underwent laparotomy with resection of the mass. The tumor did not infiltrate adjacent organs and the surrounding desmoplastic reaction allowed negative surgical margins with prostate, bladder and rectum preservation. 


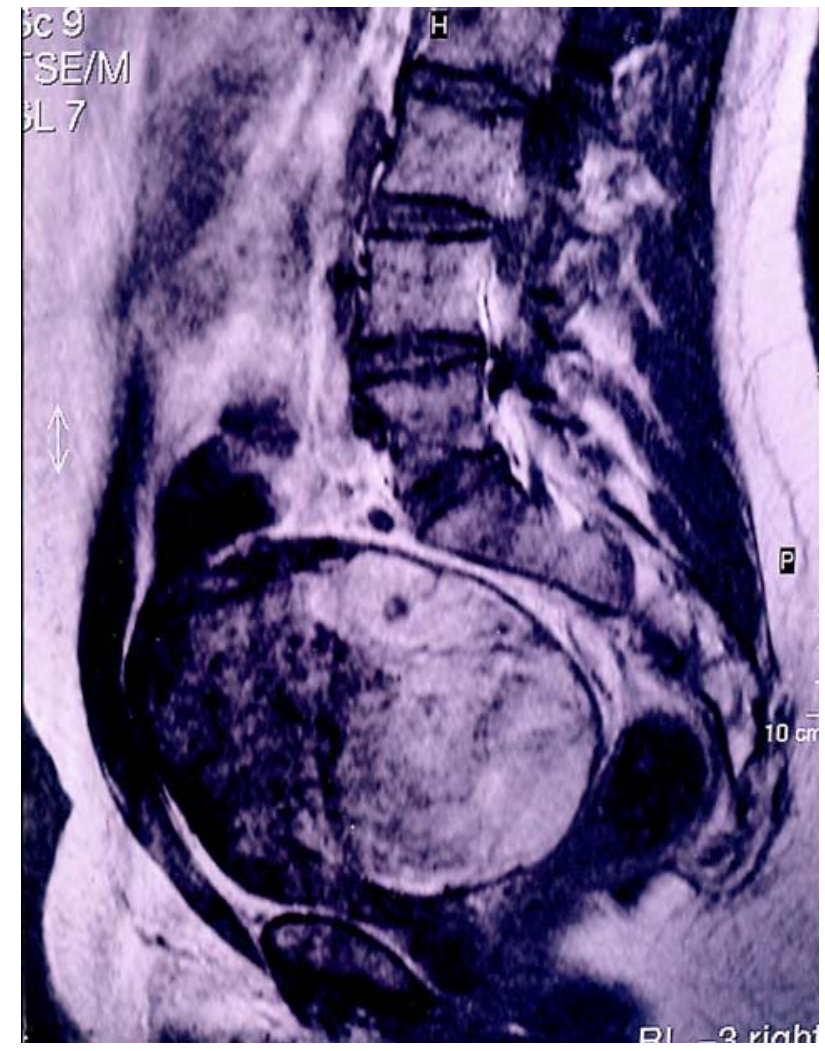

Figure 1 - Magnetic resonance imaging of the pelvis showing seminal vesicle tumor.

Histological exam showed an enormous amount of necrosis and hemorrhage intermixed by a monotonous pattern of small round blue cells with discreet pleomorfism, clear cytoplasm, solid block setting with cystic areas and fibrous colagenized stroma (Figure-2). Immunohistochemical examination was positive for CD-99 (Figure-3) and S-100 protein while muscular actin, gp100 antigen, CD34, Desmin, Melan A/MART-1, EWS-FLI1, Alpha-Inibin and Calreatin were negative. Focally positive staining for cytokeratin 40, 48, 50 and 50.6 KDa was found. The combination of the histological and immunohistochemical results leaded to the diagnosis of a PNET. Reciprocal translocation $\mathrm{t}(11 ; 22)(\mathrm{q} 24 ; \mathrm{q} 12)$ using transcriptase-reverse Polymerase Chain Reaction (RTPCR) was inconclusive in the present study due to the lack of PGK1 and ACBT genes amplification of the surgical specimen.

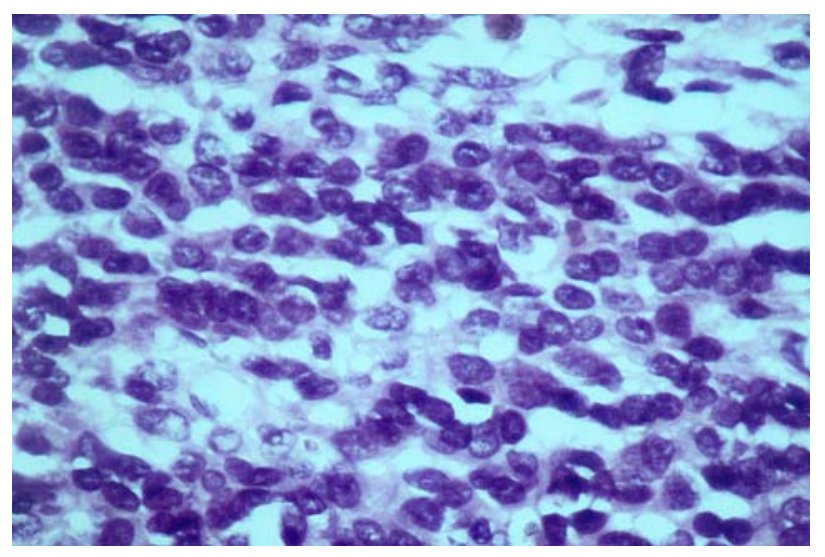

Figure 2 - Histological analysis showing a monotonous pattern of small round cells (HE, X400).

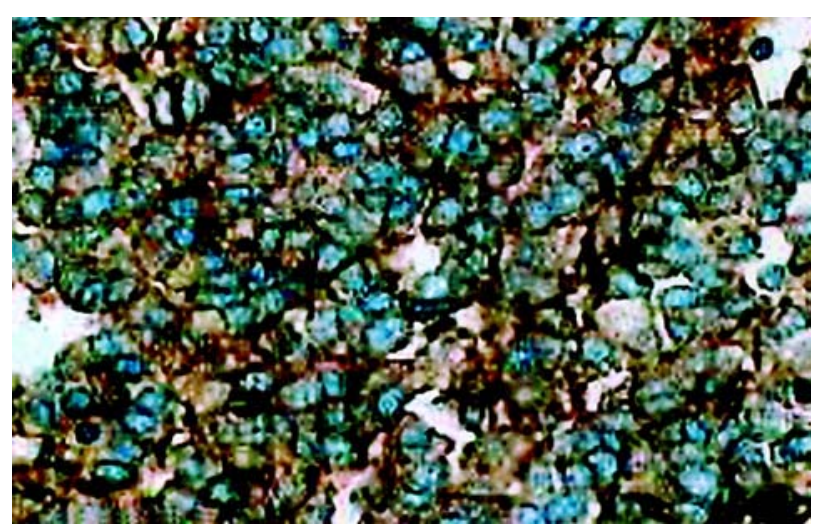

Figure 3 - CD99 positivity (Immunohistochemical staining, X100).

After a three years follow up, the patient remained potent, with normal urinary and digestive functions and showed no relapse.

\section{COMMENTS}

Genitourinary origin of PNET is rare and diagnosis is difficult and usually confused with primary rectum or prostate carcinomas, benign tumors (1) or with other small round blue cell tumors (SRBCT). Differential diagnosis must include Ewing's sarcomas, peripheral medulloepithelioma, rhabdomyosarcomas, myxoid liposarcomas, malignant fibrous his- 
tiocytomas chondrosarcomas, lymphoblastic lymphoma and even small cell osteosarcomas and neuroblastomas. Differential diagnosis must also include benign conditions like prostatic utricle cyst, prostatic abscess, seminal vesicle hydrops, seminal vesicle cyst, seminal vesicle empyema, ectopic ureterocele, fibrous obturator fossa cyst, hemangiopericytoma, among others (2).

Some genitourinary PNETs have already been reported arising from kidney, adrenal gland, prostate, spermatic cord and testis, but to the best of our knowledge, up to now, no seminal vesicle origin has been published.

Although PNETs can be accurately diagnosed using time-honored morphologic criteria and immunohistochemistry, the genetic confirmation of the translocation $\mathrm{t}(11 ; 22)(\mathrm{q} 24 ; \mathrm{q} 12)$ by cytogenetics and/ or molecular analysis is essential for the diagnosis of unusual morphologic variants, including adamantinoma-like, spindled, esclerosis and clear cell/ anaplastic variants (3). CD99 expresses protein product of the fusion gene EWS/FLI-1 and are often positive in PNET, SRBCT and Ewing's sarcoma family. The combination of histological, immunohistochemical and, sometimes, cytogenetics leads the final diagnosis. In the present case, despite the lack of cytogenetic confirmation of the reciprocal translocation $t$ $(11 ; 22)$ (q24;q12), the positivity of CD99 and S-100 protein in the immunohistochemical exam and the histological presentation favored PNET diagnosis.

The biological behavior of this tumor is expressed by a rapid growing mass non-responsive to chemotherapy and frequently associated to distant metastasis. The presence of an extensive area of necrosis in histopathological exam means that there might have been an expressive response to the neoadjuvant chemotherapy, which was clinically reflected by a favorable evolution regarding relapse and survival.

Surgical approach is the best treatment modality and new chemotherapy agents are necessary to achieve better results in metastatic disease.

\section{CONFLICT OF INTEREST}

None declared.

\section{REFERENCES}

1. Martinez Ibanez V, Abad P, Toran N, Gonzalez CI, Sanchez de Toledo J, Marques A, et al.: Primitive neuroectodermal tumors: difficult tumors versus modern oncology. Cir Pediatr. 1998; 11: 5-9.

2. Dahms SE, Hohenfellner M, Linn JF, Eggersmann C, Haupt G, Thuroff JW: Retrovesical mass in men: pitfalls of differential diagnosis. J Urol. 1999; 161: 12448.

3. Folpe AL, Goldblum JR, Rubin BP, Shehata BM, Liu W, Dei Tos AP, et al.: Morphologic and immunophenotypic diversity in Ewing family tumors: a study of 66 genetically confirmed cases. Am J Surg Pathol. 2005; 29: 1025-33. 


\section{EDITORIAL COMMENT}

Primary seminal vesical tumors either benign or malignant are very rare. Primary adenocarcinoma of the seminal vesicle is a rare tumor. Only 50 cases have been reported in literature. Differential diagnosis includes carcinoma extending from the urinary bladder, prostate, or rectum. Cystadenomas may be incidentally discovered on rectal examination or at autopsy in middleaged men, or the patients may present hemospermia or nonspecific suprapubic pain. Epithelial-stromal tumors of the seminal vesicle include a spectrum of tumors that grossly and microscopically resemble the similarly named tumor of the prostate, and fibroadenoma and phyllodes tumor of the breast. The distinction between cystadenoma and epithelial-stromal tumor of the seminal vesicle is based on the microscopic aspect of the stroma: normal or possibly reactive smooth muscle favors cystadenoma and hypercellular, presumably neoplastic stroma favors epithelial-stromal tumor.

Other tumors are equally very rare. The present report is a unique case of primary small round undifferentiated tumor of the seminal vesicle. There is a spectrum of tumors showing small round undifferentiated cells including $\mathrm{T}$ lymphoblastic lymphomas, poorly differentiated synovial sarcomas, some neuroendocrine carcinomas, rare cases of rhabdomyosarcoma usually of alveolar type and the primitive neuroectodermal tumors (PNET). The latter having been the source of both controversy and rapid advances in recent years but the term PNET is now the preferred term to describe a family of lesions that are characterized by a specific and reproducible reciprocal chromosome translocation, $\mathrm{t}(11 ; 22)(\mathrm{q} 24 ; \mathrm{q} 12)$. These tumors show morphologic, immunohistochemical, ultrastructural and tissue cultural evidence of neuroendocrine differentiation. In the present case, in spite of lack of cytogenetic confirmation of the reciprocal translocation, the positivity of CD99 and S-100 protein in the immunohistochemical exam and the histological features favor the diagnosis of PNET.

Dr. Athanase Billis

Full-Professor of Pathology State University of Campinas, Unicamp

Campinas, São Paulo, Brazil E-mail: athanase@fcm.unicamp.br 